\title{
Vol. 65, No. 18
}

In the report, "Progress Toward Polio Eradication Worldwide, 2015-2016," on page 471, in Table 2, the title should have read "Number of reported poliovirus cases, by country - worldwide, January 1, 2015-May 4, 2016," and the heading for the second column should have been "2015 (January-December).” 